\title{
Distúrbios osteomusculares e o trabalho dos que cuidam de idosos institucionalizados
}

\section{Musculoskeletal disorders and the care work of elderly in institutions}

\author{
Maria do Carmo Baracho de Alencar ${ }^{[a]}$, Vanessa Mann Schultze ${ }^{[b]}$, Sandra Dias de Souza ${ }^{[c]}$ \\ [a] Professora Doutora do Departamento de Ciências da Saúde da Universidade Federal de São Paulo (Unifesp), São Paulo, SP \\ - Brasil, e-mail: alencar@unifesp.br \\ [b] Acadêmica do curso de Fisioterapia da Universidade Tuiuti do Paraná (UTP), bolsista (PIBIC), Curitiba, PR - Brasil, e-mail: \\ vanessaschultze@gmail.com \\ [c] Professora Mestre do curso de Fisioterapia da Universidade Tuiuti do Paraná (UTP), Curitiba, PR - Brasil, e-mail: \\ sandias@terra.com.br
}

\section{Resumo}

Introdução: Os distúrbios osteomusculares relacionados ao trabalho continuam comuns entre trabalhadores, e permanece a preocupação dentro do tema "Promoção à saúde no trabalho". Objetivo: Investigar, em instituições na cidade de Curitiba, PR, as relações existentes entre as desordens osteomusculares de trabalhadores que cuidam de idosos, as condições de trabalho e a capacidade funcional dos idosos. Materiais e métodos: Consistiram de elaboração de questionário contendo: dados demográficos, questões relacionadas ao trabalho, dificuldades no trabalho, entre outras; e os instrumentos: Questionário Nórdico de Sintomas Osteomusculares (QNSO), com questões sobre a dor lombar, e aplicação do Índice de Barthel nos idosos atendidos pelos trabalhadores. Os questionários foram aplicados sob forma de entrevista e posteriormente ocorreram levantamentos das tarefas e observações sistemáticas das atividades, com base na Ergonomia. Resultados: Participaram 43 trabalhadores que cuidam de idosos, de ambos os gêneros e de 14 instituições; e 308 idosos por eles assistidos. A profissão dos trabalhadores variou entre cuidadores de idosos, auxiliares de enfermagem, auxiliares de serviços gerais e limpeza. Foram encontrados sintomas de dores osteomusculares nos últimos 30 dias em $62,7 \%$ dos trabalhadores, sendo as regiões das dores mais prevalentes: lombar, cervical, ombros e joelhos; e 90,0\% apresentaram um único emprego. As atividades de trabalho de maior dificuldade foram: troca de fraldas e transferências posturais. Em alguns locais, o ritmo de trabalho, pressão temporal e pequena quantidade de funcionários para as demandas exigidas, também influenciaram na presença de dores músculo-esqueléticas. Conclusão: Concluiu-se que fatores relacionados à organização do trabalho influenciaram nas desordens osteomusculares.

Palavras-chave: DORT. Ergonomia. Cuidadores. 


\begin{abstract}
Introduction: The musculoskeletal disorders still common next to workers and still a worry next to the theme of health promotion at work. Objective: Investigate the relationship in between musculoskeletal disorders in workers that take care of elderly, conditions of work, and functional capacity of elderly, in institutions of Curitiba, PR. Materials and methods: Consisted of elaborating a questionnaire containing: demographic data, questions related to work, difficulties at work, and others; and the instruments Nordic Questionnaire for Musculoskeletal Disorders -QNSO, questions related to low back pain, and application of Barthel Index in the elderly attended by the workers. The questionnaires were applied as interview, and after occurred analyses of the tasks and systematic observations of activities, with Ergonomics basis. Results: Participated 43 workers, both sexes that take care of 308 elderly assisted by them, in 14 institutions. The profession varied in people that take care of elderly, nurses' assistant, general services' assistance, and cleaners' assistant. Found musculoskeletal pain in the last thirty days in 62,7\% of workers, and with prevalence in regions: low back, cervical, shoulders and knees; and 90,0\% of the workers had only one employ. The work activities of major difficulties were: changing diaper, and postural changes. Also in some places the rhythm of work, temporal pressure, and low number of functionaries for de demands exegete, influenced in the presence of the pain. Conclusion: The study concluded that factors related to work organization influenced in the musculoskeletal disorders.
\end{abstract}

Keywords: WRMSD. Ergonomics. Care workers.

\title{
Introdução
}

Os distúrbios osteomusculares ou músculo-esqueléticos relacionados ao trabalho (DORT) incluem uma variedade de condições inflamatórias e degenerativas afetando os músculos, tendões, ligamentos, articulações, nervos periféricos, etc; incluindo: inflamações em tendões (tendinites), tenossinuvites, bursites, compressões nervosas (como síndrome do túnel do carpo, dor ciática), bem como outras condições como mialgias, lombalgias, etc. (1). Não ocorrem por uma única causa e os quadros clínicos são em geral relacionados ao sistema músculo-esquelético submetido a determinadas condições de trabalho. Estudos trazem como fatores de risco para o surgimento de DORTs: utilização de força muscular, repetição de movimentos, posturas estáticas prolongadas, sendo esses fatores associados às dimensões intensidade, velocidade, exposição temporal, entre outros $(2,3)$.

Os distúrbios do sistema músculo-esquelético no trabalho ocorrem, frequentemente, quando a demanda física do trabalho excede a capacidade física do trabalhador, e a diferença de massa muscular, composição corporal e tamanho das mulheres em relação aos homens, pode representar, para o gênero feminino, um fator de risco predisponente da sintomatologia dolorosa (4). Há também um desgaste adicional para a maioria das mulheres, pelo fato de permanecerem com a responsabilidade de cuidado com a casa e família (5). As queixas de dores músculo-esqueléticas aumentam muitas vezes por causa da dupla jornada de trabalho à qual o trabalhador é submetido, exercendo atividades que exigem determinado esforço físico, em posturas inadequadas ou tarefas repetitivas que acarretam grande desgaste físico e mental, tendo seu rendimento prejudicado pela instauração do processo de fadiga muscular e mental (6).

A Organização Mundial de Saúde (7) caracterizou as doenças músculo-esqueléticas relacionadas ao trabalho como multifatoriais, envolvendo fatores físicos, organizacionais, psicossociais, aspectos individuais e socioculturais. Uma atenção especial tem sido dada atualmente aos aspectos da Organização do Trabalho, na promoção à saúde no trabalho. A Organização do Trabalho influencia o planejamento, execução e avaliação das etapas do processo de trabalho, prescrevendo regras, normas e alguns parâmetros que norteiam o trabalho em geral. $\mathrm{O}$ trabalho vem se moldando às novas configurações de realidade e 
sociedade e muitas vezes é associado ao controle gerencial do processo aliado a uma hierarquia rígida, que tem levado os trabalhadores à insatisfação e desmotivação; e ainda a desequilíbrios nas cargas de trabalho. Sob condições adversas, essas sobrecargas podem afetar os sistemas físico e psicológico. Wisner (8) cita que a carga de trabalho está diretamente relacionada às exigências do trabalho e às formas de enfrentamento do trabalhador. Em situações adversas no trabalho, o trabalhador tentará se adaptar às situações, buscando estratégias de defesa contra os conflitos e constrangimentos, e o único componente sobre o qual o trabalhador age é sobre seu próprio corpo (físico e mental). Para Colombini e Occhipinti (9), entre os aspectos da organização do trabalho e o surgimento dos DORTs estão o caráter repetitivo das tarefas, ritmo intenso de trabalho, ausência de treinamentos, horas extras de trabalho, entre outros.

Alguns modelos de gestão acabam impondo aos trabalhadores exigências físicas e mentais no trabalho, resultando em ambientes de trabalho tensos (no sentido de haver conflitos interpessoais, insatisfação, entre outros), podendo influenciar o surgimento de sintomas dolorosos. Segundo Couto (10), a tensão muscular excessiva compromete a nutrição dos músculos, mesmo durante o repouso, levando ao acúmulo de ácido lático, que é um potente irritante das terminações nervosas da dor; assim, situações de trabalho muito tensas podem resultar em dor muscular, além de fadiga excessiva. Além disso, aspectos psicossociais são também associados aos DORTs (11-13), estando entre os aspectos: pressão no trabalho, pouca influência no trabalho, pouca capacidade de abertura, trabalho monótono, pobre relacionamento interpessoal, entre outros.

Uma das profissões que lida com idosos e apresenta riscos de desenvolver distúrbios ou desordens osteomusculares relacionadas ao trabalho é a do enfermeiro. No trabalho de enfermeiras, as profissionais estão expostas não só ao trabalho físico pesado que as tarefas exigem, mas também a um grande número de eventos, circunstâncias e condições (demandas do trabalho, transferências e movimentações de pacientes e de objetos pesados, tomada de decisões, conflitos no trabalho, entre outros) que requerem ação ou resposta (14). Em um estudo realizado com os trabalhadores de enfermagem, as regiões dolorosas mais atingidas foram: região lombar, ombros, cervical e joelhos; sendo a região lombar a mais acometida (15). A dor lombar é um dos maiores problemas de saúde pública e tem grande prevalência em enfermeiras e auxiliares $(16,17)$.

Para Alexandre et al. (18), quando se avaliam as lesões em coluna vertebral, devem ser analisados o relacionamento da dor com algum tipo de esforço físico, postura, movimento, tensão emocional, entre outros. Em outro estudo, as lesões ocupacionais que afetam diretamente a coluna vertebral ocorreram com maior frequência com os atendentes de enfermagem, no próprio quarto do paciente, principalmente durante a manipulação de equipamentos e pacientes obesos e dependentes, sendo os pisos escorregadios o principal agente causador de quedas (19).

Sobre a postura corporal dos trabalhadores de enfermagem durante a movimentação de pacientes acamados, Zanon et al. (20) evidenciaram que a postura corporal mais frequentemente assumida foi a coluna inclinada e pernas em extensão de joelhos. Como cita Cheng (21), a inclinação para a frente sem apoios adequados de membros superiores proporciona sobrecargas em vértebras lombares. Os trabalhadores de enfermagem apontaram os aspectos: pequeno espaço, elevada carga física e falta de pessoal como as principais dificuldades encontradas na execução da movimentação do paciente no leito (19). Radovanic et al. (22) ressaltam que os trabalhadores de enfermagem devem sempre avaliar as circunstâncias dos pacientes como: peso, altura, consciência, nível de dependência (mobilidade, transferência) e também os acessórios e equipamentos conectados ao paciente; sendo, para Alexandre et al. (23), importante que a movimentação e o transporte de obesos sejam minuciosamente avaliados e planejados, usando sempre que possível auxílios mecânicos. As restrições mais frequentes para os profissionais de enfermagem foram relacionadas aos aspectos ergonômicos (24), cabendo ressaltar que o estudo concentrou-se no domínio de especialidade da ergonomia física.

Os distúrbios osteomusculares relacionados ao trabalho continuam crescendo entre a população de trabalhadores, e o problema torna-se cada vez mais preocupante dentro do cenário mundial de promoção à saúde (6). O objetivo deste estudo foi investigar relações existentes entre as desordens osteomusculares de trabalhadores que cuidam de idosos em instituições, condições de trabalho, e níveis de dependência funcional dos idosos. 


\section{Materiais e métodos}

O estudo foi do tipo descritivo, com dados quantitativos e qualitativos, e a pesquisa consistiu de duas etapas. Na primeira etapa, após revisões bibliográficas sobre o tema, foi elaborado um questionário composto de perguntas sobre dados demográficos, tais como: nome, gênero, idade, níveis de escolaridade; e perguntas relacionadas ao trabalho, tais como: profissão atual, tempo de atuação ( 0 a 5 meses, 6 meses a 2 anos e mais de 2 anos), se atuou no cuidado ao idoso anteriormente, se trabalhava em outro local, tempo de trabalho - horas/dia - $(0$ a 2 horas, 3 a 6 horas, 7 a 8 horas, 9 a 10 horas e mais que 10 horas), se tem folga no trabalho, percepção do esforço durante o trabalho (nenhum, leve, moderado, elevado), número de idosos assistidos por dia (1 a 2, 3 a 4, 5 a 6,6 a 7 e mais que 8 idosos), se realizava outras atividades no trabalho (limpar e arrumar, cozinhar, lavar roupa, passar roupa, outros), se faziam transferências posturais dos idosos sozinhos (sempre, às vezes e nunca), e questões semiabertas sobre as dificuldades encontradas no trabalho. Ainda no questionário, os instrumentos de pesquisa: Questionário Nórdico de Sintomas Osteomusculares (QNSO), validado no Brasil (25), baseado na versão original do Questionário Nórdico Musculoesquelético (QNM) (26). $\mathrm{O}$ instrumento permite a identificação de desordens osteomusculares e fornece descrições quanto à ocorrência de sintomas (dor, desconforto ou dormência) nas várias regiões anatômicas (pescoço, ombros, coluna - região torácica e lombar, cotovelos, punhos e mãos, quadril, joelho, tornozelo e pé). As variáveis dependentes são as áreas de desconforto para cada região, intensidade de desconforto nos últimos 12 meses, presença de desconforto nos últimos 7 e 30 dias, e afastamento do trabalho nos últimos 12 meses. O índice de severidade dos sintomas variam de 0 a 4 , sendo que 0 representa ausência de sintomas. Em outro instrumento de avaliação da coluna vertebral (18), foram utilizadas apenas as seguintes questões: em relação à dor, incluindo a escala analógica da dor em relação à dor lombar (na qual 0 representa ausência de dor e 10 corresponde à maior dor que já sentiu); classificação do estágio da dor lombar em: aguda (duração de 1 a 4 semanas), sub-aguda (duração de 5 a 12 semanas), crônica (duração de mais de 12 semanas) e crônica com exacerbação aguda; quando sente dor (ao acordar, à tarde, final do dia, sem horário fixo) e fatores que pioram a dor lombar (esforço físico, repouso prolongado, posição em pé, posição sentada, esporte, deambulação, ao movimento de segmentos corporais, tensão emocional).

Também foi utilizado um instrumento para avaliação da capacidade funcional dos idosos (atendidos pelos trabalhadores), o Índice de Barthel (27). Esse índice analisa dez atividades: alimentação, transferências, toalete pessoal, uso de banheiro, tomar banho, andar em superfície plana, subir e descer escadas, vestir-se e despir-se; na pontuação proposta por Barthel, valores (score) $\geq 80 \geq 100$ indicam que o indivíduo é independente, score entre $60<80$ indicam leve dependência, score de $>40<60$ indicam moderada dependência; sendo que valores maiores ou iguais a 60 indicam alta probabilidade de o indivíduo continuar a viver na comunidade, e score $\leq 40$ são considerados com grande dependência.

Foram selecionadas 14 instituições aleatoriamente, conforme disponibilidade e interesse em participar da pesquisa. A seleção dos trabalhadores teve como critério de inclusão um contato direto com o idoso (cuidado) nas atividades de trabalho, e foram avaliados todos os indivíduos que trabalhavam em contato direto com os idosos nas instituições no turno/dia, com exceção de enfermeiros que apresentavam rotinas de trabalho diferenciadas e exerciam também tarefas administrativas. O projeto foi aprovado pelo Comitê de Ética da Universidade Tuiuti do Paraná (n. 093/07), e os entrevistados foram informados sobre a pesquisa e assinaram um Termo de Consentimento Livre e Esclarecido. 
$\mathrm{Na}$ segunda etapa, houve levantamentos das tarefas e ocorreram observações sistemáticas das atividades de trabalho, com base na Ergonomia, e aplicação do questionário sob forma de entrevista, e anotações (caneta e papel) de relatos espontâneos dos trabalhadores nas suas atividades de trabalho. A entrevista com cada trabalhador levou em média 50-60 minutos, com permissão das Instituições para sua realização. Além da entrevista com o trabalhador, foram também realizados análises das capacidades funcionais dos idosos (Índice de Barthel) atendidos, sob forma de entrevista com o idoso, e na impossibilidade de obter a resposta do idoso, os dados foram obtidos do trabalhador, que também conferia os dados quando respondido pelo idoso, posteriormente.

Os dados quantitativos foram analisados estatisticamente usando o Sistema SAS®.

\section{Resultados e discussão}

Participaram do estudo 43 trabalhadores de ambos os sexos (97,7\% mulheres e 2,3\% homens) que cuidavam de idosos em 14 instituições da região metropolitana de Curitiba, PR, e avaliados 308 idosos (capacidade funcional pelo Índice de Barthel). Para Fernandes et al. (28), há um número maior de mulheres no trabalho de enfermagem por ser o cuidado seu objeto de trabalho, caracterizado como feminino pelas representações sociais. A faixa etária dos trabalhadores variou entre 0 a 20 anos (7,0\%); de 21 a 40 anos, a maioria dos trabalhadores (67,4\%); e entre 41 e 60 anos (23,3\%). O tempo de serviço era: até cinco meses (30,2\%); de 6 meses a dois anos $(37,2 \%)$; e mais de dois anos $(37,2 \%)$.

A escolaridade dos trabalhadores variou de $1^{\circ}$ grau incompleto $(20,9 \%) ; 1^{\circ}$ grau completo $(9,3 \%) ; 2^{\circ}$ incompleto $(7,0 \%) ; 2^{\circ}$ grau completo $(55,8 \%)$; e $3^{\circ}$ grau entre incompleto e completo $(7,0 \%)$. A profissão dos trabalhadores (conforme registro em carteira) se dividiu da seguinte forma: $44,2 \%$ cuidadores de idosos; 34,9\% auxiliares de enfermagem; 18,6\% auxiliares de serviços gerais; 2,3\% auxiliares de limpeza. Foram encontrados alguns enfermeiros (em pouca quantidade) e em alguns locais não havia a presença deste profissional contratado (instituições menores); esses profissionais eram responsáveis pela supervisão dos demais trabalhadores que cuidavam dos idosos, pelos curativos e controle dos medicamentos e outras tarefas administrativas. Dos que participaram do estudo, 53,5\% nunca haviam, até então, trabalhado com idosos, sem relatos de treinamentos específicos prévios, e trabalhavam apenas em um local 90,7\% dos entrevistados, alguns tinham dois empregos, e alguns, que moravam no próprio local de trabalho, relataram a dificuldade de não se envolver com os problemas em horários livres, tendo que sempre "ajudar no serviço".

Dos entrevistados, $58,2 \%$ trabalhavam mais que 10 horas por dia, em dias alternados (escala 12 x 36 horas). Em relação à percepção do esforço físico durante o trabalho, 76,7\% consideraram de moderado à elevado. As tarefas gerais dos trabalhadores nas instituições eram: dar auxílio ao banho, alimentação (café da manhã), auxiliar na vestimenta, trocar fraldas, alimentação (almoço), higiene oral, alimentação (lanche e jantar), e colocar os idosos para dormir. Sendo as atividades de trabalho de maior dificuldade entre os trabalhadores a troca de fraldas, banho, e transferências posturais (envolvendo as transferências posturais cadeira de rodas-cama e vice-versa, cadeira de rodas-cadeira de banho e viceversa). Dos trabalhadores, $83,7 \%$ cuidavam de mais de oito idosos por dia, sendo que 55,8\% realizavam outras tarefas como: limpeza do asilo, lavar roupas, cozinhar, passar roupas, entre outras.

Do total de 43 trabalhadores, 62,7\% tiveram alguma dor osteomuscular no último mês, a prevalência e regiões acometidas estão demonstradas no Gráfico 1: 


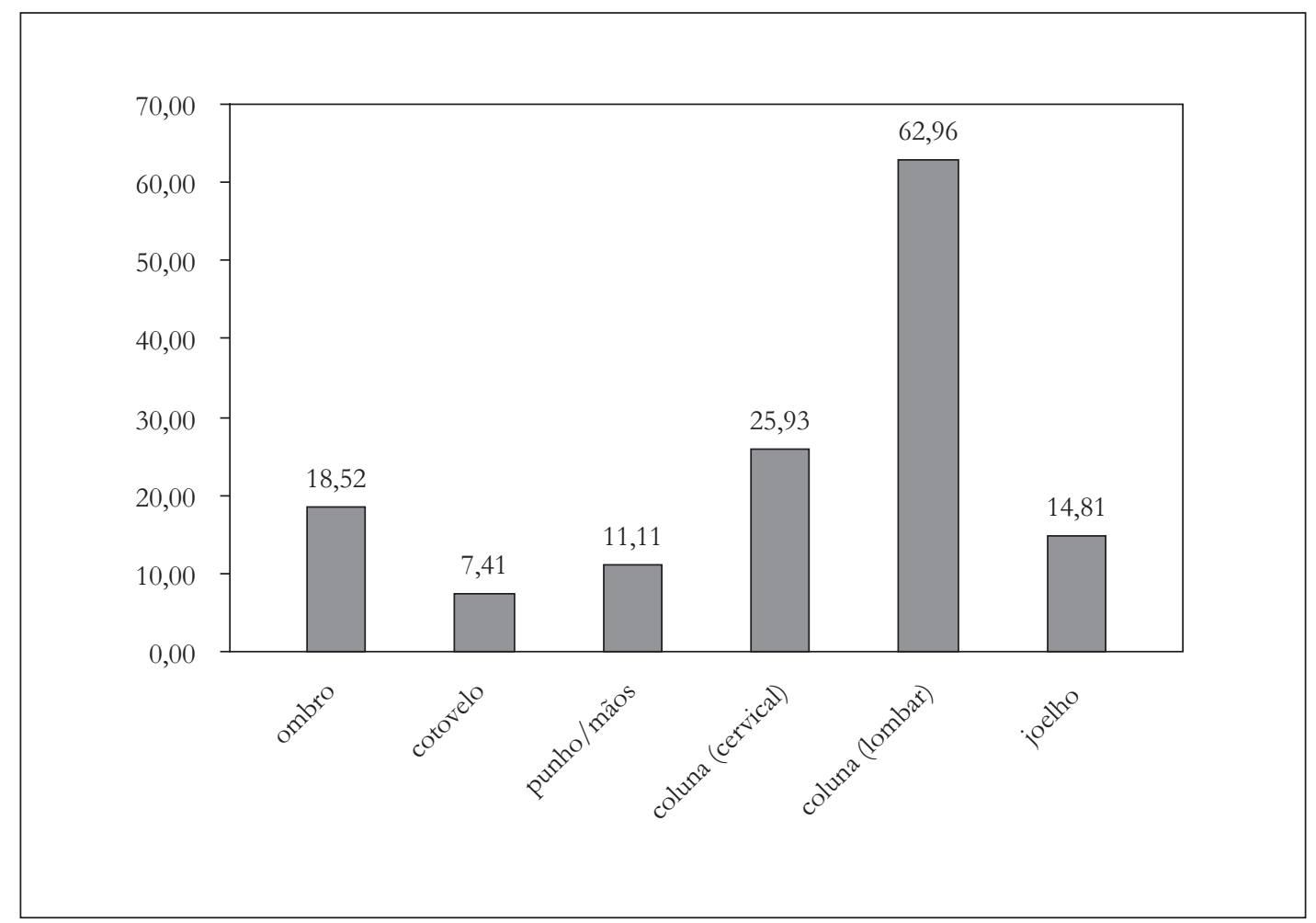

Gráfico 1 - Prevalência e regiões das dores osteomusculares dos trabalhadores nos últimos 30 dias (valores em porcentagem)

Os sintomas (dor/desconforto) foram 62,9\% em região lombar, 25,9\% em região cervical, $18,5 \%$ em ombros, entre outras; com alguns trabalhadores apresentando mais de uma região dolorosa (n =12). Sobre o fato de haver mais de uma região dolorosa em alguns trabalhadores, sugere-se que esteja associado a outros aspectos ergonômicos, como altura da cama do idoso, altura de locais onde colocam sabonetes e xampu no banho, de condições de cadeiras de roda nos transportes, entre outros relacionados a outras tarefas exigidas (limpeza do local, lavar roupas, etc.). O estudo de Gurgueira et al. (15) encontrou como as regiões anatômicas mais atingidas em trabalhadores de enfermagem a região lombar, ombros, cervical e joelhos; apesar deste estudo apresentar a maioria dos trabalhadores da categoria de cuidadores de idosos (função em carteira) há algumas características similares regionais quanto às dores. Não houve associação estatística significativa entre a idade e a presença de dor osteomuscular entre os avaliados. O tempo de serviço entre os entrevistados foi de $67,4 \%$, com no máximo dois anos de trabalho, indicando alta rotatividade no trabalho, o que sugere insatisfação no trabalho.

Em relação às transferências posturais, alguns trabalhadores evitavam de as executar sozinhos, porém, 53,5\% relataram realizar, às vezes, essa atividade sozinhos pelo fato de não terem colegas disponíveis para ajudar, com exigências no sentido de ter que dar conta das tarefas, conforme relatos. O nível de dependência do idoso, especialmente relacionado ao aspecto motor, influenciou o esforço físico necessário para algumas atividades. Um dos relatos expressa uma condição de trabalho: "peguei sozinha uma [idosa] que tinha uns $100 \mathrm{~kg}$, e fiquei com dor nas costas", e acrescentou: "o esforço que a gente faz é muito grande"; quanto às dores uma trabalhadora revela: "depois que comecei a trabalhar aqui, a dor vai e vem..", e outra profissional declara: "quando eu venho trabalhar, tenho dor nas costas", indicando a influência de processos de trabalho na sintomatologia dolorosa. Além disso, pelo fato de o trabalho exigir muitas tarefas, os trabalhadores têm pouco tempo para dedicar maior dedicação às tarefas mais específicas do cuidado, como apontado por outra trabalhadora: "aqui eu corro o dia todo...", e outra complementa: "trabalhar com poucos funcionários, a gente acaba se desgastando." Não foram observados cuidados posturais, especialmente na troca de fraldas, atividade na qual foi observada a flexão do tronco e extensão de joelhos. Segundo Bongers et al. (29), a pressão do tempo acelera os movimentos e o estresse eleva a 
intensidade da contração muscular, aumentando, dessa forma, a sensibilidade à dor. Descuidos posturais, por exemplo, podem sofrer influências de aspectos relacionados à organização do trabalho.

Houve associação significativa entre o número de idosos atendidos por trabalhador (de 8 a mais) e a presença de dor osteomuscular (Teste de Fischer, com p = 0,042); contudo, sugere-se que outras análises sejam feitas, levando-se em conta aspectos relacionados aos níveis de dependência funcional dos idosos, entre outros; dados difíceis de serem analisados no estudo, pelo fato de mais de um trabalhador cuidar de mais de um idoso, e haver variáveis em relação ao quanto ele é cuidado pelo mesmo trabalhador por dia; além disso, o idoso pode apresentar déficits cognitivos ou doença mental, associados ou não ao déficit funcional motor. Dentre os trabalhadores com dores, 70,5\% deles apresentaram dor em intensidade acima de cinco no ato da pesquisa. O estágio da dor lombar mais prevalente encontrou-se em 58,8\%, com características de dor crônica (duração de mais de 12 semanas), 23,5\% aguda (duração de 1-4 semanas) e 17,6\% sub-aguda (duração de 5-12 semanas). Ainda, uma das trabalhadoras havia sido afastada nos últimos 12 meses por causa de dores osteomusculares.

\section{Em relação à capacidade funcional dos idosos}

Foram avaliados pelo Índice de Barthel um total de 308 idosos de ambos os sexos, atendidos pelos trabalhadores entrevistados, conforme demonstrado na Tabela 1:

Tabela 1 - Número de trabalhadores e de idosos nos locais e níveis de dependência

\begin{tabular}{ccccccc}
\hline LOCAL & $\begin{array}{c}\mathbf{N}^{\circ} \\
\text { TRABALHADORES }\end{array}$ & $\begin{array}{c}\mathbf{N}^{\circ} \\
\text { IDOSOS }\end{array}$ & $\begin{array}{c}\text { SCORE } \\
\mathbf{2 8 0} \geq \mathbf{1 0 0}\end{array}$ & $\begin{array}{c}\text { SCORE } \\
\mathbf{2 6 0}<\mathbf{8 0}\end{array}$ & $\begin{array}{c}\text { SCORE } \\
\mathbf{2} \mathbf{4 0}<\mathbf{6 0}\end{array}$ & $\begin{array}{c}\text { SCORE } \\
<\mathbf{4 0}\end{array}$ \\
\hline 1 & 3 & 28 & 14 & 7 & 1 & 6 \\
2 & 1 & 8 & 6 & - & 1 & 1 \\
3 & 5 & 21 & 2 & 4 & 4 & 11 \\
4 & 2 & 7 & 1 & - & - & 6 \\
5 & 3 & 28 & 8 & 3 & 1 & 16 \\
6 & 3 & 17 & 15 & 1 & - & 1 \\
7 & 1 & 6 & 6 & - & - & - \\
8 & 4 & 11 & 6 & - & 1 & 4 \\
9 & 2 & 9 & 6 & 2 & - & 1 \\
10 & 8 & 32 & 7 & 1 & 4 & 20 \\
11 & 5 & 33 & 27 & 1 & 5 & - \\
12 & 2 & 27 & 16 & 3 & 4 & 4 \\
13 & 3 & 79 & 61 & 5 & 1 & 12 \\
14 & 1 & 2 & 1 & 1 & - & - \\
\hline
\end{tabular}

A Tabela 1 apresenta o número de trabalhadores que cuidam de idosos em cada instituição; bem como a quantidade de idosos e os scores obtidos conforme o Índice de Barthel. Observaram-se variações existentes nos locais quanto à relação número de idosos, especialmente com moderada dependência e grande dependência, e de trabalhadores que os cuidavam; sugerindo, em alguns casos, proporção inadequada, especialmente em relação às exigências (sobrecargas físicas), como observado especialmente nos locais 13 e 5, nos quais havia maior número de idosos com grande dependência. 
As dificuldades encontradas em relação ao trabalho, neste estudo, foram na atividade de troca de fraldas e nas transferências posturais (como cadeira de rodas-cama e vice-versa, cadeira de rodas-cadeira de banho e vice-versa). Esses dados vão de encontro aos do estudo de Radovanic et al. (22), que demonstra que as maiores dificuldades apresentadas pelos trabalhadores estiveram relacionadas às transferências e na movimentação dos pacientes. Contudo, neste estudo outras dificuldades também surgiram entre alguns trabalhadores e estiveram associadas aos relacionamentos interpessoais (entre equipe e entre o idoso) e excesso de tarefas, aliado ao número reduzido de funcionários. Alguns idosos de dependência moderada poderiam auxiliar os trabalhadores em algumas transferências posturais, se houvesse mais tempo para isso, em algumas situações, a demanda para o trabalho era grande, e o trabalhador acabava por executar as atividades como se o mesmo tivesse grande dependência e não pudesse auxiliar; ter paciência é uma necessidade para o trabalho. Em relação ao relacionamento com os idosos, os trabalhadores apresentaram diferentes comportamentos e havia uma relação emocional entre o trabalhador e o idoso. O trabalho que envolve o cuidar estabelece algum vínculo afetivo, o qual causa desgaste pela tensão gerada entre envolver-se afetivamente e não completar o circuito afetivo, e por sentirem-se incapazes de modificar as situações que se lhe apresentam (30). Algumas situações podem dificultar o trabalho, como observado na fala de um trabalhador: "ontem foi estressante, tem uma idosa muito grossa...", e como observado também em outro relato: "tem idosas que xingam a gente, que falam palavrão...". Adequações dos modos operatórios na atividade de trabalho sofrem influências na prática de outras sobrecargas a que os trabalhadores estão submetidos.

\section{Conclusão}

Concluiu-se com este estudo que a troca de fraldas e as transferências posturais foram atividades de maiores dificuldades entre os trabalhadores. As regiões das dores osteomusculares mais prevalentes entre os trabalhadores foram: lombar, cervical, ombros e joelhos. Em alguns locais, aspectos organizacionais como: ritmo de trabalho, pressão temporal na realização das atividades, poucos funcionários para as demandas exigidas e ausência de treinamentos influenciaram a presença das dores osteomusculares dos trabalhadores; a maioria dos trabalhadores tinha um único emprego. Outro aspecto encontrado em alguns locais foi: pobre relacionamento interpessoal e exigências de outras tarefas para alguns trabalhadores, além das relacionadas ao cuidado dos idosos.

Sugerimos com este estudo que as instituições determinem um número máximo de idosos a serem cuidados pelo trabalhador e, conforme o nível de capacidade funcional do idoso, que haja reorganização e melhora na distribuição de tarefas e processos de trabalho, na tentativa de promover melhorias nas condições de trabalho, além de oferecer treinamentos específicos. Sempre que possível, propor ações interdisciplinares que promovam maior integração entre a equipe de um modo geral.

Pesquisas futuras devem ser realizadas a fim de investigar essas questões e suas relações com as desordens osteomusculares.

\section{Referências}

1. Punnet L, Wegman DH. Work related musculoskeletal disorders: the epidemiologic evidence and debate. J Electromyogr Kinesiol. 2004;14(1):13-23.

2. Kumar S. Theories of musculoskeletal injury causation. Ergonomics. 2001;44(1):17-47.

3. Marras WS. Occupational low back disorder: causation and control. Ergonomics. 2000;43(7):880-902.

4. Maciel ACC, Fernandes MB, Medeiros LS. Prevalência e fatores associados à sintomatologia dolorosa entre profissionais da indústria têxtil. Rev Bras Epidemiolog. 2006;9(1):94-102. 
5. Rocha LE, Debert-Ribeiro M. Trabalho, saúde e gênero: estudo comparativo sobre analistas de sistemas. Rev Saúde Pública. 2001;35(6):539-47.

6. Brandão AG, horta BL, Tomasi E. Sintomas de distúrbios osteomusculares em bancários de Pelotas e região: prevalência e fatores associados. Rev Bras Epidemiolog. 2005;8(3):295-305.

7. Rio RP. LER- ciência e lei. Belo Horizonte: Health; 1998.

8. Wisner A. A inteligência do trabalho: textos selecionados em ergonomia. São Paulo: Fundacentro; 1994.

9. Colombini D, Occhipinti E. Preventing upper limb work-related musculoskeletal disorders (UL-WRMSD): new approaches in job (re) design and current trends in standadization. Appl Ergon. 2006;37:441-50.

10. Couto HA. Ergonomia aplicada ao trabalho: o manual da máquina humana. Belo Horizonte: Ergo; 1996.

11. Davies KG, Heaney CA. The relationship between psychosocial work characteristics and low back pain: underlying methodological issues. Clin Biomech. 2000;15(6):389-406.

12. Feng CK, Chen ML, Mao IF. Prevalence of and risk factors for different measures of low back pain among female nursing aides in Taiwanese nursing homes. BMC Musculoskeletal Disorders. Serial in internet, 2007 (cited 2008 feb 14). Available from: www.biomedcentral.com/1471-274/8/52

13. Smith DR, Mihashi M, Adachi Y, Koga H, Ishitake TA. A detailed analyses of musculoskeletal disorder risk factors among Japanese nurses. J Safety Res. 2006;37(2):195-200.

14. Eriksen W, Bruusgaard D, Knardahl S. Work factors of sickness absence: a three month prospective study of nurses'aides. Occup Environ Med. 2003;60:271-8.

15. Gurgueira GP, Alexandre NMC, Corrêa Filho HR. Prevalência de sintomas músculo-esquelético em trabalhadoras de enfermagem. Rev Latino-am Enfermagem. 2003;11(5):608-13.

16. Hartvigsen J, Lauritzen S, Lings S, Lauritzen T. Intensive education combined with low tech ergonomic intervention does not prevent low back pain in nurses. Occup Environ Med. 2005;62(1):13-7.

17. Horneij EL, Jensen IB, Holmströn EB, Ekdahl C. Sick leave among home-care personnel: a longitudinal study of risk factors. BMC Musculoskelet Disord. 2004;5(1):38.

18. Alexandre NMC, Moraes MAA. Modelo de avaliação físico-funcional da coluna vertebral. Rev Latino-am Enfermagem. 2001;9(2):67-75.

19. Parada EO, Alexandre NMC, Benatti MCC. Lesões ocupacionais afetando a coluna vertebral em trabalhadores de enfermagem. Rev Latino-am Enfermagem. 2002;10(1):64-9.

20. Zanon E, Marziale MHP. Avaliação da postura corporal dos trabalhadores de enfermagem na movimentação de pacientes acamados. Rev Esc Enf USP. 2000;34(1):26-36.

21. Chen YL. Predicting the vertebral inclination of lumbar spine. Ergonomics. 2000;43(6):744-51.

22. Radovanic CAT, Alexandre NMC. Validation of an instrument for patient handling assessment. Appl Ergon. 2004;35(4):321-8.

23. Alexandre NMC, Rogante MM. Movimentação e transferência de pacientes: aspectos posturais e ergonômicos. Rev Esc Enf USP. 2000;34(2):165-73.

24. Gurgueira GP, Alexandre NMC. Laudos médicos recomendando restrições de trabalho em um hospital universitário no Brasil. Rev Latino-am Enfermagem. [periódico online]. 2006 [capturado em 25 out. 2009];14(4). Disponível em: http://www.scielo.br/pdf/rlae/v14n4/pt_v14n4a07.pdf

25. Pinheiro FA, Tróccoli BT, Carvel CV. Validação do questionário nórdico de sintomas osteomusculares como medida de morbidade. Rev Saúde Pública. 2002;36:307-12. 
26. Kuoringa I, Jonsson B, Kilbom A, Vinterberg H, Biering-Sorensen F, Anderson G, et al. Standardised nordic questionnaires for analyses of musculoskeletal symptoms. Appl Ergon. 1987;18:233-237.

27. Mahoney FI, Barthel D. Functional evaluation: the Barthel index. Md State Med J. 1965;14:61-5.

28. Fernandes JD, Ferreira SL, Albergaria AK, Conceição FM. Saúde mental e trabalho feminino: imagens e representações de enfermeiras. Rev. Latino-am Enfermagem. 2002;10(2):199-206.

29. Bongers PM, Winter CR, Kompier MAJ, Ildebrandt VH. Psychosocial factors at work and musculoskeletal disease. Scandinavian Journal of Work Environment Health. 1993;19:297-312.

30. Vasques-Menezes I. Saúde mental e trabalho: aplicações na prática clínica. In: Jacques MG, Codo W. Saúde mental e trabalho: leituras. Petrópolis: Vozes; 2002.

Recebido: $28 / 11 / 2008$

Received: $11 / 28 / 2008$

Aprovado: 02/09/2009

Approved: 09/02/2009 